\title{
Distribution of Loss Severity of Bonus-Malus Systems
}

\author{
Maryam Teimourian ${ }^{1 *}$, Mohammad Q. Vahidi-As/ ${ }^{2}$ and Amir T. Payandeh Najafabadi ${ }^{2}$ \\ 'Department of Statistics, College of Science, Tehran Science and Research Branch, \\ Islamic Azad University, Tehran, Iran; teimorymar@gmail.com \\ ${ }^{2}$ Department of Statistics, Shahid Beheshti University, G. C. Evin, Tehran, Iran; \\ m-vahidi@sbu.ac.ir, Amirtpayandeh@sbu.ac.ir
}

\begin{abstract}
This paper describes a method for computing the distribution function of the loss severity in a Bonus-Malus system with $k$ levels. A closed form expression for the distribution function of the loss severity by assuming different distributions for claim sizes and claim numbers in each level has been derived. Also, Ruin probability and computing VaR in the system have been given.
\end{abstract}

Keywords: Bonus-Malus System, Cauchy Residue, Characteristic Function, Distribution of Loss Severity

\section{Introduction}

Bonus-Malus system is one of non-life actuarial model. In fact, bonuses can be earned by not filing claims, and a malus is incurred whenever any claim has been filed. For more information see Denuit et al. ${ }^{5}$. In practice, a BonusMalus scale consists of finite number of levels, numbered from 1 to $k$, each with its own relative premium. So, the portfolio of an insurance company can be classified into $k$ different groups as policyholders in the first group have the smallest risk-tendency and the policyholder in the last group have the largest risk-tendency in the portfolio. Hence, the loss severity of the portfolio at given time $t$ is

$$
W(t)=\sum_{j=1}^{k} \sum_{l=1}^{N_{j}(t)} U_{j l}
$$

and $W(t)=0$ whenever $\sum_{j=1}^{k} N_{j}(t)=0$. The random variable $N_{j}(t)$ is the number of claims of $j^{\text {th }}$ type of contract till $t$ and $U_{j l}$ for $j=1,2, \ldots, k$ and $l=1,2, . ., N_{j}(t)$ is the size of $l^{\text {th }}$ claim of $j^{\text {th }}$ type contract.

In practice, the pure premium is the expected cost of all the claims that the policyholder will file during the coverage period. So Computing or approximating the distribution function of the loss severity with $k$ different groups of risk, i.e. $W(t)$, has been one of the central points in insurance mathematics. Besides, numerical methods like recursions or numerical inversion of Fourier transforms are becoming more important and produce excellent results for the case of a finite range of values ${ }^{10}$. Many authors have tried to derive a general method for computing loss severity distribution. A review of this can be found in Sundt ${ }^{11}$. In many practices, we need to compute loss severity distribution where the claim number process, $N(t)$, consists of two or more different processes that individually follow a specific process but with different parameter values. For example, in motor insurance one might like distinguish between male and female car owners; or the insurer may use layers in the age structure of his insured drivers. In general, one assumes that the claims come from a heterogeneous group of policyholders.

Within this framework, many papers deal with the loss severity model. Namely, Li and Garrido ${ }^{8}$ considered a classical risk process along with a generalized Erlang (2) risk process and calculate the ruin probability for a risk process. Lv et al. ${ }^{9}$ considered a risk model with two kinds of independent claim number processes which consists of a Poisson process and an ordinary renewal process and obtained an exponential upper bound for the ruin probability. Yuen et al. ${ }^{13}$ considered a bivariate compound Poisson model for two dependent classes of an insurance

${ }^{*}$ Author for correspondence 
business and used a bivariate compound binomial model to approximate the survival probability and found some bounds for the ruin probability. Guo et al. ${ }^{7}$ considered a risk process with two dependent classes, using a twodimensional Cox risk model. They investigated this process by considering two individual processes separately and derived an upper bound for the ruin probability. Dong ${ }^{6}$ studied a Sparre-Anderson negative risk sums model and proved that we can transform a risk process involving two dependent renewal processes into a risk process involving three independent renewal processes. Furthermore, they obtained integro-differential equations with some boundary conditions for the survival probability. Wang and Yuen ${ }^{12}$ considered a risk model with some dependent classes classified into groups of insurance businesses. By assuming the existence of moment generating function of aggregate claim, they found upper bounds for the ruin probability under certain assumptions. In all these works only special cases have been considered and no attempt has been made to derive loss severity distribution and ruin probability has been the main aim. But in many situations such as in computing risk measure, we need the loss severity distribution. The main purpose of this paper is to consider a loss severity model with $k$ dependent classes of claims and to derive some useful results in this direction.

In the next section we study some preliminaries. Section 3 is devoted to formulating and proving the main theorem and computing the loss severity distribution. Applications of the loss severity distribution including the computation of ruin probability and Value-at-Risk in a Bonus-Malus system are given in Section 4 .

\section{Preliminaries}

We consider a Bonus-Malus system with $k$ levels as a portfolio consisting of $k$ different types of contracts. Hence $k$ types of processes exist for the number of claim processes and $k$ different distribution for the severity of loss in each group. The loss severity of the portfolio till time $t$ is given by Equation (1). We assumed that the random variables $U_{j l}$ for $l=1,2, . ., N_{j}(t)$ are independent and identically distributed with common distribution $F_{U_{j}}, j=1,2, \ldots, k$. It is clear that the loss severity is in general a random sum of random variables. Depending on the type of portfolio, the insurer can make different assumptions on the claims number processes. The Poisson process has some good properties but in this paper we will assume any reasonable process for the claim number process. The distribution function of the loss severity of the portfolio up to time $t$ is

$$
F_{W}(w)=P(W(t) \leq w)=P\left(\sum_{j=1}^{k} \sum_{l=1}^{N_{j}(t)} U_{j l} \leq w\right) .
$$

It is most often assumed that the processes $N_{j}(t)$ and $U_{j l}$ are stochastically independent, and we usually will follow this practice. Denote the characteristic function of $W(t)$ for any positive $s$ by $\phi_{W}(s)=\int e^{i s w} d F_{W}(w)$. Hence

$$
\begin{aligned}
\phi_{W}(s) & =E\left[\left(E\left(\exp \left\{i s U_{11}\right\}\right)^{N_{1}(t)} \ldots\left(E\left(\exp \left\{i s U_{k 1}\right\}\right)^{N_{k}(t)} \mid N(t)\right]\right.\right. \\
& =g_{\mathbf{N}(\mathbf{t})}\left(\phi_{U_{1}}(s), \ldots, \phi_{U_{k}}(s)\right),
\end{aligned}
$$

where $g_{\mathrm{N}}(s)$ is the probability generating function of random vector $\mathrm{N}(t)=\left(N_{1}(t), \ldots, N_{k}(t)\right.$, and $\phi_{U_{j j}}$, $j=1,2, \ldots, k, l=1,2, . ., N_{j}(t)$, is the characteristic function of the random variable $U_{j l}$. The following lemma, from Athreya and Lahiri ${ }^{2}$, will be used frequently in the sequel. The integrability of $\phi_{W}$ in the Riemann sense is the only sufficient condition we need later.

LEMMA 1: Suppose $W$ be a random variable with the characteristic function $\phi_{W}$ that is integrable in the Riemann sense. Then $F_{W}$ is absolutely continuous with density given by

$$
f(w)=\frac{1}{2 \pi} \int_{-\infty}^{\infty} \exp \{-i s w\} \phi_{W}(s) d s
$$

Calculating the integral in (3) is not an easy task and its calculation is of great interest in this paper. We use characteristic function by inverse Fourier transform and we need to consider contour integration.

The following from Ablowitz and Fokas ${ }^{1}$ represents residue theorem and Jordan lemma.

Lemma 2: Let $\mathrm{h}(z)$ be analytic inside and on a simple closed contour $C$, except for a finite number of isolated singular points $z_{1}, z_{2}, \ldots, z_{n}$ located inside $C$. Further, suppose $C_{R}$, is a large semicircle on the circular $C$, and $|\mathrm{h}(z)| \leq K_{R}$, where $K_{R}$ depends only on $R$ and $K_{R} \rightarrow 0$ as $R \rightarrow \infty$. Then for all $k \neq 0$,

$$
\lim _{R \rightarrow \infty} \int_{C_{R}} \exp \{i k z\} \mathrm{h}(z) d z=0
$$

and therefore, 


$$
\int_{-\infty}^{\infty} \exp \{i k x\} \mathrm{h}(x) d x=2 \pi i \sum_{l=1}^{n} \operatorname{Res}\left(\exp \{\operatorname{ikz}\} \mathrm{h}(z) ; z_{l}\right)
$$

Finally, the following lemma makes available use of Jordan lemma for characteristic function of the loss severity of the portfolio.

LEMMA 3: Let $W$ be a random variable with absolutely continuous cumulative distribution function $F_{W}$ and density function $f_{W}$. For the complex function $g(\mathrm{z})$ defined by $g(\mathrm{z})=\int \exp \{i w z\} \mathrm{f}_{W}(\mathrm{w}) d w, z=x+i y$, we have

$$
\lim _{|z| \rightarrow \infty}|g(z)|=0 .
$$

Proof: For any fixed positive $\epsilon$ and each density function $f_{W}$ there exists a step function $f_{\epsilon}(w)=\sum_{m=1}^{M} c_{m} I_{\left(a_{m}, b_{m}\right)}(w)$ with $1 \leq M<+\infty$ and $a_{m} \leq b_{m}, c_{m} \in \mathbb{R}$ for $m=1, \ldots, M$, such that $\int\left|f_{W}(w)-f_{\epsilon}(w)\right| d w<\frac{\epsilon}{2}$. For $|z| \neq 0, w \in \mathbb{R}$,

$$
\begin{aligned}
|g(\mathrm{z})|= & \left|\int \exp (\mathrm{iwz}) f_{W}(w) d w\right|<\int\left|f_{w}(w)-f_{\epsilon}(w)\right| d w \\
& +\left|\int \exp (\mathrm{iwz}) f_{\epsilon}(w) d w\right| .
\end{aligned}
$$

On the other hand,

$$
\begin{aligned}
\left|\int \exp \{i z w\} f_{\epsilon}(w) d w\right|= & \left|\sum_{m=1}^{M} c_{m} \int_{a_{m}}^{b_{m}} \exp \{i z w\} d w\right| \\
& \leq \sum_{m=1}^{M} c_{m}\left|\frac{\exp \left\{(i x+y)\left(b_{m}-a_{m}\right)\right\}}{i x+y}\right|,
\end{aligned}
$$

hence for all $|z|>\left|z_{\epsilon}\right|$, with $z_{\epsilon}=4 \sum_{m=1}^{M} c_{m} \frac{\exp \left\{y\left(a_{m}-b_{m}\right)\right.}{\epsilon}$, we have $\left|\int \exp (\operatorname{iz} w) f_{\epsilon}(w) d w\right|<\frac{\epsilon}{2}$.

The next section explores some situations for computing the loss severity using Lemma 3.

\section{Theoretical Findings}

To identify a method for computing the loss severity distribution of a portfolio is one of the main purposes in insurance mathematics. For that purpose, we introduce a method that uses the previous lemmas to tackle the problem.

Theorem: Suppose $\phi_{W}$ be the characteristic function of a random variable is given by Equation (1). Where $\phi_{W}$ is integrable in the Riemann sense and $z_{l}, \ldots, z_{n}$ be singular points of complex function $\phi_{W}(z), z \in \mathcal{C}$ Then $F_{W}$ is absolutely continuous with density given by

$$
\begin{aligned}
f(w) & =\frac{1}{2 \pi} \int_{-\infty}^{\infty} e^{-i s w} \phi_{W}(s) d t \\
& \left.=i \sum_{l=1}^{n} \operatorname{Res}\left(e^{-i z w} g_{N(t)}\left(\phi_{U_{11}}(z), \ldots, \phi_{U_{k 1}}(z)\right)\right) ; z_{l}\right) .
\end{aligned}
$$

Proof: If $\phi_{W}(s)$ is integrable in the Riemann sense, using Equation (3):

$$
f(w)=\frac{1}{2 \pi} \int_{-\infty}^{\infty} e^{-i s w} \phi_{W}(s) d s .
$$

Now, we arrive at the desired result using Lemmas 2 and 3 with $K_{R}=\varepsilon$. Hence from Equation (4)

$$
\begin{aligned}
f(w) & =i \sum_{l=1}^{n} \operatorname{Res}\left(e^{-i z w} \phi_{W}(z) ; z_{l}\right) \\
& \left.=i \sum_{l=1}^{n} \operatorname{Res}\left(e^{-i z w} g_{N(t)}\left(\phi_{U_{11}}(z), \ldots, \phi_{U_{k 1}}(z)\right)\right) ; z_{l}\right),
\end{aligned}
$$

where $z_{l}, l=1, n$ are singular points of the complex function $\phi_{W}(z)$.

In the following example, we derive the distribution function of the loss severity using this theorem.

EXAMPLE: Suppose that for positive fixed $t$, $U_{1 j}, U_{2 j}, \ldots, U_{N_{j}(t) j}$ is a sequence of i.i.d random variables with a common exponential distribution

$$
f_{U_{l j}}(u)=\lambda_{j} \exp \left\{\lambda_{j} u\right\},
$$

where $j=1,2, \ldots, k ; l=1,2, \ldots, N_{j}(t)$. Then using Equation (2)

$$
\phi_{W}(s)=\sum_{n=\left(n_{1}, \ldots, n_{k}\right)} P\left(\mathbf{N}(t)=\left(n_{1}, \ldots, n_{k}\right)\right) \prod_{j=1}^{k}\left(\frac{\lambda_{j}}{\lambda_{j}-i s}\right)^{n_{j}}
$$

The characteristic function is integrable for $n_{j}>1$, and isolated singular points of $\phi_{W}(s)$ are $z_{1}=-i \lambda_{1}, \ldots, z_{k}=-i \lambda_{k}$. Therefore using Equation (5)

$$
\begin{aligned}
f(w)= & \sum_{n=\left(n_{1}, \ldots, n_{k}\right)} P\left(\mathbf{N}(t)=\left(n_{1}, \ldots, n_{k}\right)\right) \\
& \sum_{l=1}^{m} \operatorname{Res}\left(e^{-i z w} \prod_{j=1}^{k}\left(\frac{\lambda_{j}}{\lambda_{j}-i z}\right)^{n_{j}}, z=z_{l}\right) \\
= & \sum_{n=\left(n_{1}, \ldots, n_{k}\right)} P\left(\mathbf{N}(t)=\left(n_{1}, \ldots, n_{k}\right)\right) \\
& \left.\sum_{l=1}^{k} \frac{\lambda_{l}^{n_{l}} \frac{\partial^{n_{l}-1}}{\partial z^{n_{l}-1}} \prod_{j=1, j \neq l}^{k}\left(\frac{\lambda_{j}}{\lambda_{j}-i z}\right)^{n_{j}} e^{-i z w}}{\left(n_{l}-1\right) !}\right|_{z=-i \lambda_{l}} .
\end{aligned}
$$


The last relation can be computed easily and hence we arrive at the loss severity distribution of the portfolio.

\section{Application to Bonus-Malus systems}

In many situations, the loss severity distribution is needed and the above method allows us to obtain some valuable results in risk theory. Tow simple of those is given in the following. Both results, driven by Theorem, are tasks of importance in risk theory. The first one is computation the ruin probability of a Bonus-Malus system and the other one is about a risk measure.

\subsection{Ruin Probability of a Bonus-Malus System}

Let the system include $k$ levels and $M(t)$ is the number of policyholders in the system till $t$. Also, the distribution of premium of $l^{\text {th }}$ policyholder in the system is

$$
P\left(C_{l}=c\right)=\sum_{j=1}^{k} p_{j} I_{c_{j}}(c) \quad j=1,2, \ldots, k ; l=1,2, . ., M(t)
$$

where $I_{c_{j}}(c)$ is the Indicator function. The loss severity by considering $k$ dependent groups of risks is given by Equation (1). If $u_{0}$ be initial capital, the surplus process of Bonus-Malus system is

$$
\xi(\mathrm{t})=\mathrm{u}_{0}+\sum_{l=1}^{M(t)} C_{l}-\mathrm{W}(\mathrm{t})=\mathrm{u}_{0}+R(t)
$$

We want to derive the ruin probability, i.e. $P(\xi(t)<0)$ at given $t>0$. For this, we use the characteristic function of $R(t)=\sum_{l=1}^{M(t)} C_{l}-\mathrm{W}(\mathrm{t})$, the discrepancy between the total premium and aggregate claim, that is

$$
\phi_{R(t)}(s)=g_{M(t)}\left(\phi_{C}(s)\right) g_{N(t)}\left(\phi_{U_{11}}(-s), \ldots, \phi_{U_{k 1}}(-s)\right) .
$$

The following corollary introduces a relation for computing the ruin probability in Bonus-Malus systems.

Corollary 1: Suppose $\phi_{R(t)}$, the characteristic function of $R(t)$, is integrable in the Riemann sense and $z_{l}, \ldots, z_{n}$ be singular points of complex function $\phi_{R(t)}(z), z \in \mathcal{C}$. Then

$$
\begin{aligned}
& P(\xi(t)<0) \\
& =i \int^{u_{0}} \sum_{i=1}^{n} \operatorname{Re} s\left(e^{-i z r} g_{M(t)}\left(\phi_{C}(z)\right) g_{N(t)}\left(\phi_{U_{11}}(-z), \ldots, \phi_{U_{k 1}}(-z)\right) ; z_{i}\right) d r .
\end{aligned}
$$

\subsection{Value-at-Risk}

A risk measure is a mapping from the set of random variables, risks, to the real line. The purpose is to give a single value for the degree of risk or uncertainty associated with a random variable. Value at risk $(\mathrm{VaR})$ is a very popular risk management tool in many different types of organizations ${ }^{3}$. VaR by definition $\operatorname{VaR}[X ; p]=F_{X}^{-1}(p)$, has many advantages including that VaR always exits (see Denuit et al. ${ }^{4}$ ). The distribution function of loss severity is used for deriving VaR and without the distribution function of loss severity it cannot be computed.

Corollary 2: Let $\phi_{W}$ be the characteristic function of loss severity $W$. If $\phi_{W}$ is integrable with respect to the Lebesgue measure on $\mathbb{R}$ with singular points $z_{l}, \ldots, z_{n}$, then VaR is obtain from

$$
\int_{-\infty}^{V a R} \sum_{i=1}^{n} \operatorname{Res}\left(e^{-i z w} \phi_{W}(z) ; z_{i}\right) d t=p
$$

\section{Conclusions}

This article employs a well known complex variable method to provide a closed form for the distribution of loss severity of Bonus-Malus systems. We considered the system as a portfolio with $k$ dependent (or independent) classes of claim processes and different claim size distributions. A closed form for distribution function of the loss severity of such portfolio has been derived. Also, some useful results in risk theory have been given.

\section{References}

1. Ablowitz M, Fokas A. Complex Variables, Introductions and Applications. Second edition. UK, Cambridge University Press; 2003.

2. Athreya K, Lahiri S. Measure Theory and Probability Theory. New York, Springer; 2006.

3. Bali T. An extreme value approach to the estimating to estimating volatility and value at risk. Journal of Business. 2003; 76(1):83-108.

4. Denuit M, Dhaene J, Goovaerts M, Kaas R. Actuarial theory for dependent risks: measures, orders and models. New York, John Wiley and Sons; 2005. 
5. Denuit M, Maréchal X, Pitrebois S, Walhin J. Actuarial Modelling of Claim Counts : Risk Classification, Credibility and Bonus-Malus Systems. New York, John Wiley and Sons; 2007.

6. Dong Y. Ruin probability for correlated negative risk sums model with Erlang processes. Appl Math J Chinese Univ. 2009; 24(1):14-20.

7. Guo J, Yuen K, Zhou M. Ruin probability in Cox risk models with two dependent classes of business. Acta Mathematic Sinica, English Series. 2006; 23(7):1281-88.

8. Li S, Garrido J. Ruin probability for two classes of riskprocess. Astin Bulletin. 2005; 35(1):61-77.

9. Lv T, Guo J, Zhang X. Ruin probability for risk model with two classes of claims. Acta Mathematica Sinica, English Series. 2010; 26(9): 1749-60.
10. Rolski T, Schmidli H, Schmidt V, Teugels J. Stochastic processes for insurance and finance. New York, John Wiley and Sons; 1998.

11. Sundt B. Review recursive evaluation of aggregate claims distributions. Insur Math Econ. 2002; 30:297-322.

12. Wang G, Yuen K. On a correlated aggregate claims model with thinning-dependence structure. Insur Math Econ. 2005; 36:456-68.

13. Yuen $\mathrm{K}$, Guo J, Wu X. On the first time of ruin in the bivariate compound Poisson model. Insur Math Econ. 2006; 38:298-308. 